PAPER

\title{
Reduction in hypothalamic ${ }^{1} \mathrm{H}-\mathrm{MRS}$ metabolite ratios in patients with cluster headache
}

\author{
S-J Wang, J-F Lirng, J-L Fuh, J-J Chen
}

J Neurol Neurosurg Psychiatry 2006;77:622-625. doi: 10.1136/jnnp.2005.081836

See end of article for authors' affiliations .....................

Correspondence to: Dr S-J Wang, Neurological' Institute, Taipei Veterans General Hospital, Taipei, Taiwan, 112; sjwang@vghtpe.gov. tw

Received 6 October 2005 In revised form

23 November 2005 Accepted 7 December 2005

\begin{abstract}
Objective: To determine the ${ }^{1} \mathrm{H}-\mathrm{MR}$ spectroscopic (MRS) findings in the hypothalamus in patients with episodic cluster headache.

Methods: 47 patients were recruited with episodic cluster headache $(35$ in cluster period and 12 in remission), 21 normal controls, and 16 patients with chronic migraine. The hypothalamic ${ }^{1} \mathrm{H}-\mathrm{MRS}$ metabolite ratio changes in patients with cluster headache were evaluated and compared with results in the normal controls as well as patients with chronic migraine. Seven patients in the cluster period group underwent a follow up hypothalamic MRS study five to six months after remission.

Results: In patients with cluster headache, the hypothalamic $N$-acetylaspartate (NAA)/creatine $(\mathrm{Cr})$ and choline $(\mathrm{Cho}) / \mathrm{Cr}$ ratios were similar between those in cluster period and in remission. As a group, both $\mathrm{NAA} / \mathrm{Cr}$ and $\mathrm{Cho} / \mathrm{Cr}$ levels were significantly lower in patients with cluster headache in comparison with either the control or chronic migraine groups. In those with a follow up MRS study, the levels of metabolite ratios did not differ between the cluster and remission periods.

Conclusions: This study provides evidence of persistent biochemical change of the hypothalamus in patients with episodic cluster headache. Low levels of NAA/Cr and $\mathrm{Cho} / \mathrm{Cr}$ suggest that cluster headache might be related to both neuronal dysfunction and changes in the membrane lipids in the hypothalamus.
\end{abstract}

M odern neuroimaging techniques have shown that the hypothalamus is important in the pathogenesis of cluster headache. ${ }^{12}$ One study with positron emission tomography (PET) has shown activation of the ipsilateral hypothalamus during episodes of cluster headache. ${ }^{1}$ Voxel based morphometry magnetic resonance imaging has also shown bilateral structural abnormalities in the hypothalamus. ${ }^{2}$ Of note, one study reported hypothalamic dysfunction in patients with chronic migraine. ${ }^{3}$ A recent PET study also showed hypothalamic activation during episodes in patients with migraine without aura. ${ }^{4}$

Proton MR spectroscopy $\left({ }^{1} \mathrm{H}-\mathrm{MRS}\right)$ permits a non-invasive biochemical analysis of a defined volume within the brain. ${ }^{5}$ The metabolites visible in vivo using long echo ${ }^{1} \mathrm{H}$-MRS include creatine-phosphocreatine $(\mathrm{Cr})$, choline containing compounds (Cho) and $\mathrm{N}$-acetylaspartate (NAA). ${ }^{5}$ Despite the published mathematical techniques for absolute quantification, ${ }^{7}$ relative metabolite ratios, such as NAA/Cr, Cho/Cr, and NAA/Cho are valid and useful for clinical practice and research when CSF partial volume cannot be measured reliably or absolute data are not available. ${ }^{8} \mathrm{NAA} / \mathrm{Cr}$ is generally considered to be a marker of neuronal health, being reduced in conditions where there is neuronal loss or dysfunction. ${ }^{5}{ }^{10} \mathrm{Cho} / \mathrm{Cr}$ can be viewed as an indirect marker of myelination and cell membrane metabolism. ${ }^{11} 12$

Region specific decreases in $\mathrm{NAA} / \mathrm{Cr}$ or $\mathrm{Cho} / \mathrm{Cr}$ have been found in a number of different neurological conditions. Lodi et al first reported a method to detect the ${ }^{1} \mathrm{H}$-MRS in the hypothalamus and they found a decrease of $\mathrm{NAA} / \mathrm{Cr}$ in patients with narcolepsy. ${ }^{13}$ By using a similar method, this group reported in an abstract that hypothalamic NAA/Cr was decreased in patients with cluster headache. ${ }^{14}$ However, they did not report the results of the $\mathrm{Cho} / \mathrm{Cr}$ ratio or follow up the same patients during and outside the episode.

In this study, we adopted a similar method of using ${ }^{1} \mathrm{H}$ MRS to investigate the hypothalamic metabolite ratios in patients with episodic cluster headache. We compared the results in patients in cluster period or in remission with those in normal controls. A further group of patients with chronic migraine was also recruited for comparison. We also followed up the MRS study in some patients of the cluster period group when their cluster headache remitted.

\section{METHODS}

The study was designed to evaluate the change of the metabolite ratios of ${ }^{1} \mathrm{H}$-MRS in patients with episodic cluster headache. The study protocol was approved by the institution review board of the Taipei-Veterans General Hospital. All participants provided their written informed consent of participation.

\section{Subjects}

All patients were recruited from the headache clinic at the Taipei-Veterans General Hospital. Two groups of patients with episodic cluster headache were investigated, one group of patients were in their cluster period (cluster period group) and the other, during remission for at least four months (remission group). None of our participants had chronic cluster headache because patients with this diagnosis are rare in Taiwan. ${ }^{15}$ In addition, a group of normal controls and a group of patients with chronic migraine (including both probable chronic migraine and chronic migraine) were also recruited for comparisons. For normal controls, only infrequent episodic tension type headache was allowed $(<1$ headache per month). All headache diagnoses were based on the International Classification of Headache Disorders, 2nd edition (ICHD-II) (2004). ${ }^{16}$ In this study, the ages of the participants ranged between 20 and 55. In addition to group comparisons, we also followed up hypothalamic MRS in some patients in the cluster period group five to six months after remission.

Abbreviations: NAA, $\mathrm{N}$-acetylaspartate; $\mathrm{Cr}$, creatine; Cho, choline; PET, positron emission tomography; ${ }^{1} \mathrm{H}-\mathrm{MRS}$, proton MR spectroscopy; PRESS, point resolved spectroscopy; CHESS, chemical shift water suppression 


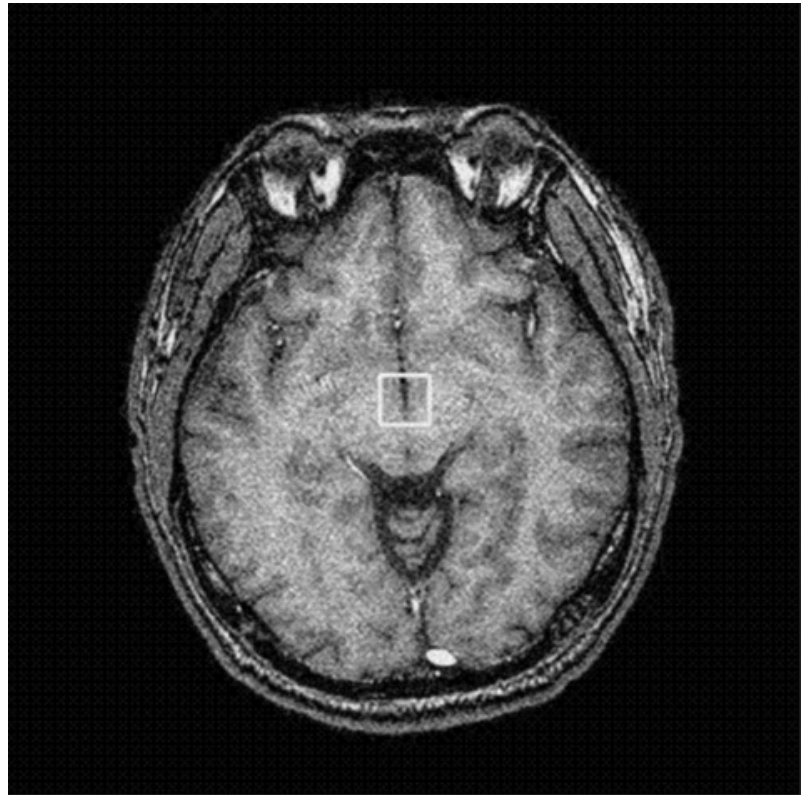

Figure 1 The VOI of MR spectroscopic study was located in bilateral hypothalami on the thin cut (1 mm) 3D fast SPGR images.

\section{Single voxel ' H-MRS of bilateral hypothalami}

We used a protocol similar to the one suggested by Lodi et al. ${ }^{14}$ ${ }^{1} \mathrm{H}$-MRS studies were performed using a 1.5 T MR unit (Signa EXCITE, GE Medical Systems, Milwaukee, WI). To obtain a higher signal to noise ratio, a $2.25 \mathrm{~cm}^{3}(1.5 \times 1.5 \times 1.0 \mathrm{~cm})$ volume of interest (VOI) was placed on axial three dimensional (3D) fast spoiled gradient recalled acquisition in steady state (SPGR) images with $1 \mathrm{~mm}$ in thickness covering bilateral hypothalami (fig 1). The VOI in each subject were placed in a uniform manner by the same investigator (JFL). Point resolved spectroscopy (PRESS) pulse sequence with a repetition time of $1500 \mathrm{~ms}$, echo time of $144 \mathrm{~ms}, 1536$ acquisitions, and 8 excitations was used for the examinations. The prescan algorithm of PROBE automatically adjusts the transmitter and receiver gains and centre frequency. The local magnetic field homogeneity is optimised with the three plane auto-shim procedure, and the flip angle of the third water suppression pulse is adjusted for chemical shift water suppression (CHESS) before PRESS acquisition. The single voxel ${ }^{1} \mathrm{H}$-MRS scan time is about 39 minutes based on the machine protocol.

Peak areas for NAA at 2.02 parts per million (ppm), Cr at $3.03 \mathrm{ppm}$, and Cho at $3.22 \mathrm{ppm}$ were calculated using the Funtool (GE XVi, Milwaukee, WI). Peak integral values were expressed relative to the $\mathrm{Cr}$ peak. Metabolite intensity ratios were automatically calculated at the end of each PROBE/SV acquisition including NAA/Cr and Cho/Cr. NAA/Cho were also calculated for comparisons. To assure the high quality, MRS results with full width at half maximum (FWHM) $>6 \mathrm{~Hz}$ were considered unqualified for MRS analyses.

\section{Statistical analysis}

As the study was to investigate the difference of the hypothalamic ${ }^{1} \mathrm{H}$-MRS metabolite ratios among different study groups, one way analysis of variance followed by post hoc Scheffe's method was used. The effects of age, sex, or clinical profile of cluster headache on metabolite ratios were tested by correlation tests or $t$ tests. In the patients of the cluster period group (who also underwent a follow up MRS after remission), the levels of metabolite ratios during and outside the bout were compared by using the paired $t$ test. All tests were two sided and $\mathrm{p}<0.05$ was considered significant.

\section{RESULTS \\ Demographic aspects}

During the study period, 106 subjects (24 F/82 M) were recruited: 58 patients with episodic cluster headache (44 in cluster period, 15 in remission ), 27 normal controls, and 20 patients with chronic migraine. Of them, 84 patients (79\%) obtained qualified MRS results. The success rates did not differ among groups. Table 1 lists the demographic aspects and clinical profiles of the final analysis groups. Age did not differ among the four study groups. Because of the nature of the diseases studied, patients with chronic migraine had a higher female ratio and patients with cluster headache had a higher male ratio. However, the sex ratio did not differ between the control and cluster headache groups. The headache profiles did not differ between the two subgroups of the cluster headache patients.

\section{${ }^{1} H$-MRS differences among different groups}

The mean (SD) levels of all ${ }^{1} \mathrm{H}$-MRS metabolite ratios were similar between cluster period and remission groups (NAA/Cr 1.69 (0.21) v 1.73 (0.11); Cho/Cr 1.67 (0.21) v 1.69 (0.22); $\mathrm{NAA} / \mathrm{Cho} 1.02(0.12) v 1.04(0.15))$. To increase the statistical power, we combined both cluster headache groups into one group for further analysis. Table 2 shows the comparisons of the mean levels of these metabolite ratios among the three groups. The differences of NAA/Cr and $\mathrm{Cho} / \mathrm{Cr}$ but not NAA/ Cho were significant among these three groups (one way analysis of variance). Pair wise comparisons by Scheffe's method showed that patients with cluster headache had lower levels of NAA/Cr and Cho/Cr than both the control and chronic migraine groups. Of note, the chronic migraine group showed similar levels of hypothalamic ${ }^{1} \mathrm{H}-\mathrm{MRS}$ metabolite ratios to those of the control group.

Table 1 Demographic aspects and headache profile of the study subjects with evaluable MRS results

\begin{tabular}{|c|c|c|c|c|}
\hline & $\begin{array}{l}\text { Normal control } \\
\text { group }(n=21)\end{array}$ & $\begin{array}{l}\text { Cluster period group } \\
(n=35)\end{array}$ & Remission group $(n=12)$ & $\begin{array}{l}\text { Chronic migraine group } \\
(\mathrm{n}=16)\end{array}$ \\
\hline Female/male (N) & $8 / 13$ & $6 / 29$ & $2 / 10$ & $12 / 4$ \\
\hline Age (y), mean (SD) (range) & $36.1(9.0)(23-51)$ & $36.7(7.7)(24-54)$ & $37.0(5.9)(25-44)$ & $42.3(10.9)(20-54)$ \\
\hline Duration of the illness (y), mean (SD) (range) & & $11.1(8.0)(0.5-30)$ & $12.8(9.1)(3-30)$ & $14.0(7.8)(1.5-27)$ \\
\hline $\begin{array}{l}\text { Total number of cluster period, mean (SD) } \\
\text { (range) }\end{array}$ & & $9.4(7.3)(1-30)$ & $9.0(8.6)(2-30)$ & \\
\hline $\begin{array}{l}\text { Duration of the current cluster period (days), } \\
\text { mean (SD) (range) }\end{array}$ & & $19.3(10.0)(2-40)$ & & \\
\hline $\begin{array}{l}\text { Duration of remission from the last cluster } \\
\text { period (months), mean (SD) (range) }\end{array}$ & & & $16.7(15.8)(4-60)$ & \\
\hline $\begin{array}{l}\text { Frequency of cluster periods (times/y), mean } \\
\text { (SD) (range) }\end{array}$ & & $1.03(0.71)(0.25-4)$ & $0.68(0.35)(0.29-1.5)$ & \\
\hline Side of cluster episodes (right/left) & & $25 / 10$ & $8 / 4$ & \\
\hline
\end{tabular}


Table 2 Comparisons of the levels of the metabolic ratios of ${ }^{1} \mathrm{H}-\mathrm{MRS}$ of the study groups

\begin{tabular}{lllll}
\hline & $\begin{array}{l}\text { Normal controls } \\
(\mathbf{n}=\mathbf{2 1})\end{array}$ & $\begin{array}{l}\text { Cluster headache } \\
(\mathbf{n}=\mathbf{4 7})\end{array}$ & $\begin{array}{l}\text { Chronic migraine } \\
(\mathbf{n}=16)\end{array}$ & p Valuet \\
\hline $\mathrm{NAA} / \mathrm{Cr}$ & $1.93(0.18)^{*}$ & $1.70(0.18)^{*} \ddagger$ & $1.85(0.28) \ddagger$ & $<0.001$ \\
$\mathrm{Cho} / \mathrm{Cr}$ & $1.85(0.34)^{*}$ & $1.67(0.21)^{*} \ddagger$ & $1.89(0.31) \ddagger$ & 0.006 \\
$\mathrm{NAA} / \mathrm{Cho}$ & $1.07(0.19)$ & $1.03(0.13)$ & $0.99(0.11)$ & 0.219 \\
\hline
\end{tabular}

\section{Clinical correlates of ${ }^{1} \mathrm{H}-\mathrm{MRS}$ in patients with cluster headache}

Except for age in relation with NAA/Cr $(r=-0.39$, $\mathrm{p}=0.008$ ), we did not find any further clinical correlates for the metabolite ratios of NAA/Cr or Cho/Cr including age at onset, sex, duration of disease, total number of cluster periods, frequency of episodes per year, duration of present cluster period in patients of the cluster period group, duration of remission in patients of the remission group, or the use of prophylactic agents for cluster headaches (data not shown).

\section{Follow up MRS results}

Ten patients $(8 \mathrm{M} / 2 \mathrm{~F})$ in the cluster period group underwent a follow up hypothalamic MRS study five to six months after remission. Seven of them $(6 \mathrm{M} / 1 \mathrm{~F}$, mean age 36.4 years) obtained qualified results. The levels of the metabolite ratios did not change significantly after remission (paired $t$ test) (table 3).

\section{DISCUSSION}

Our study showed change in ${ }^{1} \mathrm{H}$-MRS metabolite ratios in the hypothalamus in patients with episodic cluster headache, not only the NAA/Cr but also Cho/Cr. Patients during and outside the cluster periods showed similar levels of metabolite ratios. This was shown by group comparisons (cross sectional design) or comparisons from the cluster period to the remission period in the same patients (longitudinal design). In addition, our study also showed that the hypothalamic ${ }^{1} \mathrm{H}$-MRS metabolite ratios differed between patients with cluster headache and patients with chronic migraine.

${ }^{1} \mathrm{H}$-MRS is a non-invasive method to study the change of metabolites in certain brain regions for many neurological illnesses. However, the technique of hypothalamus ${ }^{1} \mathrm{H}$-MRS is more difficult because of the depth of the hypothalamus. Based on Lodi's method ${ }^{13}$ the acquisition time ( 39 minutes) is long. Even though our VOI was larger $\left(2.25 \mathrm{~cm}^{3}\right)$ than Lodi et al's $\left(1.2-1.4 \mathrm{~cm}^{3}\right),{ }^{14}$ one fifth of the study's ${ }^{1} \mathrm{H}$-MRS results could not fulfil the selection criteria. Nevertheless, our data on NAA/Cr in control and episodic cluster headache groups were similar to those reported by Lodi et al, ${ }^{14}$ suggesting the reliability of the methodology. The low NAA/ $\mathrm{Cr}$ and $\mathrm{Cho} / \mathrm{Cr}$ suggest hypothalamic neuronal and myelin dysfunction or loss in patients with episodic cluster headache. $^{5-11}$ As our follow up results were consistent with the group comparisons (cluster period $v$ remission groups), patients with episodic cluster headache probably had persistent biochemical changes in the hypothalamus, at least within five to six months to a mean of 16.7 months after remission based on our follow up study in patients of the cluster period group or those of our remission group. We hypothesise that the reduction of the hypothalamic metabolite ratios is more probably a trait (primary) rather than a state (secondary) finding and implies disease susceptibility in patients with episodic cluster headache. There is evidence that the periaqueductal grey and trigeminal nucleus caudalis are under tonic inhibition from the hypothalamus. ${ }^{17} 18$ Therefore, change of metabolites in the hypothalamus might cause disinhibition of descending input, which in turn, theoretically results in uncontrolled trigeminovascular activation, as seen in patients with cluster headache. ${ }^{18}{ }^{19}$ Of note, because the hypothalamus was scanned bilaterally, our study did not examine whether the metabolite changes in the hypothalamus were ipsilateral or bilateral in patients with cluster headache.

One study reported hypothalamic endocrinopathy in patients with chronic migraine. ${ }^{3}$ However, we found the levels of hypothalamic NAA/Cr and Cho/Cr in patients with chronic migraine were similar to those of our controls. Nevertheless, these metabolite ratio levels were different from those in patients with episodic cluster headache. When interpreting these data, keep in mind that they are ratios rather than absolute data. Furthermore, because the new ICHD-II diagnostic criteria of chronic migraine are strict and most patients were female, ${ }^{16} 20$ the chronic migraine group in our study was small and had a higher female ratio in comparison with the cluster headache group. Therefore, one should be cautious in interpreting the results. Nevertheless, we propose that different MRS patterns suggest, at a minimum, that the part played by the hypothalamus might differ between these two headache types; or, unlike cluster headache, the hypothalamus might not play a pivotal part in the pathophysiology of chronic migraine. The identification of different patterns of MRS results in the hypothalamus of different types of headache patients might eventually provide a clinical indicator. Further studies recruiting more patients are needed to clarify this point.

Some methodological issues should be discussed. Firstly, as the hypothalamus is small, bilateral hypothalami were scanned simultaneously to increase the signal to noise ratio in this study. Unlike the absolute data, the metabolite ratios used in this study were valid despite the partial volume effect of the cerebrospinal fluid. Secondly, ${ }^{1} \mathrm{H}$-MRS metabolite ratios measured in this study were subjective to the change of $\mathrm{Cr}$ contents, which was hypothesised to be stable among

Table 3 Comparisons of the levels of the ${ }^{1} \mathrm{H}-\mathrm{MRS}$ metabolic ratios during the cluster period and after remission in those with a follow up study $(n=7)$

\begin{tabular}{lllll}
\hline & During cluster period & After remission & Mean difference & p Value* \\
\hline $\mathrm{NAA} / \mathrm{Cr}$ & $1.70(0.26)$ & $1.71(0.10)$ & $-0.019(0.11)$ & 0.864 \\
$\mathrm{Cho} / \mathrm{Cr}$ & $1.79(0.32)$ & $1.80(0.32)$ & $-0.028(0.08)$ & 0.967 \\
$\mathrm{NAA} / \mathrm{Cho}$ & $0.95(0.06)$ & $0.98(0.19)$ & $-0.071(0.17)$ & 0.722 \\
\hline *Paired $t$ test. & & & \\
\hline
\end{tabular}


different study groups. This might not be true. Therefore, a quantitative method used to calculate the absolute content of these important metabolites might provide the genuine change of chemical components. ${ }^{7}$ Thirdly, our study did not recruit patients with chronic cluster headache because patients with this diagnosis are rare in Asians. ${ }^{15}$ Therefore, one should be cautious if trying to compare our study with previous studies. ${ }^{12}{ }^{14}$ In contrast, one advantage of our study was that we were able to recruit a large $(n=47)$ and homogenous group of episodic cluster headache patients. Fourthly, our follow up sample size in the cluster period group was small $(n=7)$, type II error should be considered. However, our results showed the mean differences in NAA/Cr and Cho/Cr during and outside the bout were 0.019 and 0.028 with SDs of 0.11 and 0.08 . With $80 \%$ power and a two sided probability of 0.05 for type I error, we estimate the numbers of patients required to detect a meaningful difference are around 1571 for $\mathrm{NAA} / \mathrm{Cr}$ and 13198 for Cho/Cr. Therefore, the possibility of type II error causing a negative result in this study is negligible. Fifthly, except for age (compared with $\mathrm{NAA} / \mathrm{Cr}$ ), we did not find any clinical correlates for the hypothalamic NAA/Cr or Cho/Cr ratios. Therefore, the pattern of biochemical changes of the hypothalamic ${ }^{1} \mathrm{H}-\mathrm{MRS}$ metabolites in patients with cluster headache needs further studies. Sixthly, because the ratios were examined, we repeated statistics with non-parametric methods, which yielded the same results (data not shown).

\section{Authors' affiliations}

S-J Wang, J-L Fuh, Neurological Institute, Taipei Veterans General Hospital, Taipei, Taiwan and National Yang-Ming University School of Medicine, Taipei, Taiwan

J-F Lirng, Department of Radiology, Taipei Veterans General Hospital and National Yang-Ming University School of Medicine J-J Chen, Nursing Department, Taipei Veterans General Hospital

Funding: this study was supported in part by grants from Taipei-Veterans General Hospital (V94-295)

Completing interests: none declared

\section{REFERENCES}

1 May A, Bahra A, Büchel C, et al. Hypothalamic activation in cluster headache attacks. Lancet 1998;352:275-8.

2 May A, Ashburner J, Büchel C, et al. Correlation between structural and functional changes in brain in an idiopathic headache syndrome. Nat Med 1999;5:836-8.

3 Peres MF, Sanchez del Rio M, Seabra ML, et al. Hypothalamic involvement in chronic migraine. J Neurol Neurosurg Psychiatry 2001;71:747-51.

4 Denuelle M, Fabre N, Payoux P, et al. Brainstem and hypothalamic activation in spontaneous migraine attacks: a PET study. Cephalalgia 2004;24:782.

5 Rudkin TM, Arnold DL. Proton magnetic resonance spectroscopy for the diagnosis and management of cerebral disorders. Arch Neurol 1999;56:919-26.

6 Michaelis T, Merboldt KD, Hanicke W, et al. On the identification of cerebral metabolites in localized IH NMR spectra of human brain in vivo. NMR Biomed 1991;4:90-8.

7 Provencher SW. Automatic quantitation of localized in vivo $1 \mathrm{H}$ spectra with LCModel. NMR Biomed 2001; 14:260-4.

8 Safriel Y, Pol-Rodriguez MA, Novotny EJ, et al. Reference values for long echo time MR spectroscopy in healthy adults. AJNR Am J Neuroradiol 2005;26: 1439-45.

9 Bates TE, Strangward M, Keelan J, et al. Inhibition of $\mathrm{N}$-acetylaspartate production: implications for 1H MRS studies in vivo. Neuroreport 1996;7:1397-400.

10 Davie CA, Hawkins CP, Barker GJ, et al. Serial proton magnetic resonance spectroscopy in acute multiple sclerosis lesions. Brain 1994;117:49-58.

11 Miller BL. A review of chemical issues in 1H NMR spectroscopy: N-acetyl-Laspartate, creatine and choline. NMR Biomed 1991;4:47-52.

12 Cox IJ. Development and applications of in vivo clinical magnetic resonance spectroscopy. Prog Biophys Mol Biol 1996;65:45-81.

13 Lodi R, Tonon C, Vignattelli L, et al. In vivo evidence of neuronal loss in the hypothalamus of narcoleptic patients. Neurology 2004;63:1513-15.

14 Lodi R, Pierangeli G, Tonon C. Hypothalamus metabolism studied in cluster headache patients by proton MR spectroscopy [abstract]. Ann Neurol 2004;56(suppl 8):S52.

15 Lin KH, Wang PJ, Fuh JL, et al. Cluster headache in Taiwanese-a clinicbased study. Cephalalgia 2004;24:631-8.

16 Headache Classification Subcommittee of the International Headache Society. The international classification of headache disorders. Cephalalgia 2004;24(suppl 1):1-160.

17 Swaab DF, Hofman MA, Lucassen PJ, et al. Functional neuroanatomy and neuropathology of the human hypothalamus. Anat Embryol 1993:187:317-30

18 Bartsch T, Levy MJ, Knight YE, et al. Inhibition of nociceptive dural input in the trigeminal nucleus caudalis by somatostatin receptor blockade in the posterior hypothalamus. Pain 2005;117:30-9.

19 Matharu MS, Levy MJ, Meeran K, et al. Subcutaneous octreotide in cluster headache: randomized placebo-controlled double-blind crossover study. Ann Neurol 2004;56:488-94

20 Bigal ME, Rapoport AM, Sheftell FD, et al. Chronic migraine is an earlier stage of transformed migraine in adults. Neurology 2005;65:1556-61. 